\title{
Temperature Dependence of the Viscosity of Isotropic Liquids
}

\author{
J. JADŻYN, G. Czechowski AND T. LECH \\ Institute of Molecular Physics, Polish Academy of Sciences \\ Smoluchowskiego 17, 60-179 Poznań, Poland
}

(Received November 20, 2001; in final form February 8, 2002)

\begin{abstract}
Temperature dependence of the shear viscosity measured for isotropic liquids belonging to the three homologous series: 4-(trans-4'-n-alkylcyclohexyl) isothiocyanatobenzenes $\left(\mathrm{C}_{n} \mathrm{H}_{2 n+1} \mathrm{CyHx} \quad \mathrm{Ph}\right.$ NCS; $n \mathrm{CHBT}$, $n=0 \div 12), n$-alkylcyanobiphenyls $\left(\mathrm{C}_{n} \mathrm{H}_{2 n+1} \mathrm{Ph} \mathrm{Ph} \mathrm{CN} ; n \mathrm{CB}, n=2 \div 12\right)$ and $1, n$-alkanediols $\left(\mathrm{HO}\left(\mathrm{CH}_{2}\right)_{n} \mathrm{OH} ; 1, n \mathrm{AD}, n=2 \div 10\right)$ were analysed with the use of Arrhenius equation and its two modifications: Vogel-Fulcher and proposed in this paper. The extrapolation of the isothermal viscosity of $1, n$-alkanediols ( $n=2 \div 10$ ) to $n=1$ leads to an interesting conclusion concerning the expected viscosity of methanediol, $\mathrm{HOCH}_{2} \mathrm{OH}$, the compound strongly unstable in a pure state.
\end{abstract}

PACS numbers: $66.20 .+\mathrm{d}, 83.85 . \mathrm{Jn}$

\section{Introduction}

The velocity gradient of flowing fluid produces a stream of the momentum which transfers itself towards a less-velocity part of the medium. From macroscopic point of view, the momentum transport can be quantitatively described by the shear viscosity defined as a proportionality coefficient between the shear stress and the shear rate (velocity gradient).

The viscous properties of fluids are the subject of interest of both the technicians and the scientists. It is obvious that the viscosity of a medium plays a crucial role in many technical processes and a great effort is undertaken in finding the liquids (mainly oils) of an appropriate value of the viscosity with temperature dependence as weak as possible. For thermally activated processes, as the viscous flow, a weak temperature dependence corresponds to a small value of the activation energy of the process. 
Numerous papers on the viscosity simulations have been published and an essential progress was recently achieved in understanding of the viscosity of fluids on the molecular level [1-10]. Two molecular features are considered as an essential in the phenomenon of the momentum transport in isotropic fluids: the shape of molecules and the intermolecular interactions. Studies of the influence of these two molecular features on the experimental value of the viscosity and its temperature behavior are the subject of the present paper.

\section{Experimental}

The shear viscosity was measured with a Haake viscometer RV20 with the measuring system CV100. The system consists of a rotary beaker filled with studied liquid and a cylindrical sensor of Mooney-Ewart type (M15), placed in the center of the beaker. The liquid gap was $0.5 \mathrm{~mm}$. The studied liquids show a Newtonian behavior in the available shear rates $\left(30 \div 300 \mathrm{~s}^{-1}\right)$. The accuracy of the viscosity determination was $0.5 \%$. The temperature of the sample was controlled within $\pm 0.1^{\circ} \mathrm{C}$.

Two series of the compounds studied: $n \mathrm{CHBT}$ and $n \mathrm{CB}$, mesogenic for $n \geq 4$ and $n \geq 5$, respectively, were synthesized and purified at the Institute of Chemistry, Military University of Technology, Warsaw. The purity of the compounds, minimum $99.5 \%$, was controlled by the chromatography. The viscosity data presented in this paper concern the isotropic phase of $n \mathrm{CHBT}$ and $n \mathrm{CB}$. The $1, n$-alkanediols were of commercial standard with the purity of about $98 \%$. The details concerning the phase transitions temperature of the compounds together with the numerical values of the measured viscosity are presented in [11].

\section{Results and discussion}

Figures 1a, 2a, and 3a present temperature dependences of the viscosity measured for the three series of compounds. The series are differentiated in the intermolecular interactions: from relatively weak dipolar interactions in case of $n \mathrm{CHBT}$ (the dipole moment of a single molecule $\mu_{0}$ equals to $3.5 \mathrm{D}$ ), the strong dipolar interactions in $n \mathrm{CB}\left(\mu_{0}=5 \mathrm{D}\right)$, to moderate strong hydrogen bonds $\mathrm{O}-\mathrm{H}$. . O , localized at the ends of $1, n$-diol molecules. Within each of the series, the length of the molecules is changing due to a different number $(n)$ of $-\mathrm{CH}_{2}-$ groups. Figure 4 shows, as an example, $n$ dependence of the viscosity of the studied compounds at constant temperature $\left(72^{\circ} \mathrm{C}\right)$. Two conclusions seem to be important: (i) the polarity of the molecules reflects in the viscosity of liquids as inconspicuous effect only: the $\eta$ values for $n \mathrm{CHBT}$ and $n \mathrm{CB}$ are quite close to each other; (ii) strongly nonlinear $\eta(n)$ dependence for $1, n$-diols is in contrast to the dependence observed for dipolar liquids. 

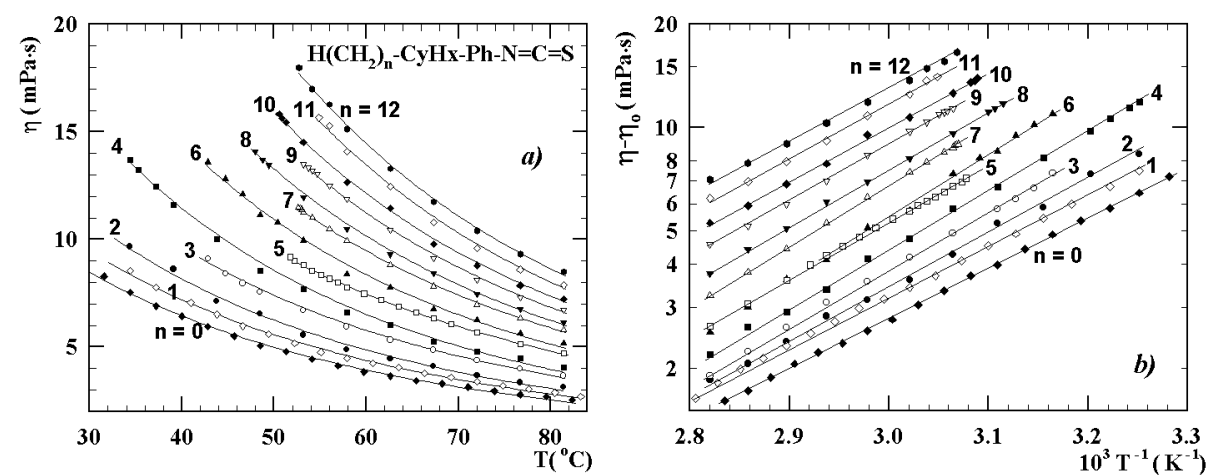

Fig. 1. Temperature dependences of the shear viscosity of $n$ CHBT homologous series (a) and Arrhenius-like plots (b) according to Eq. (3).
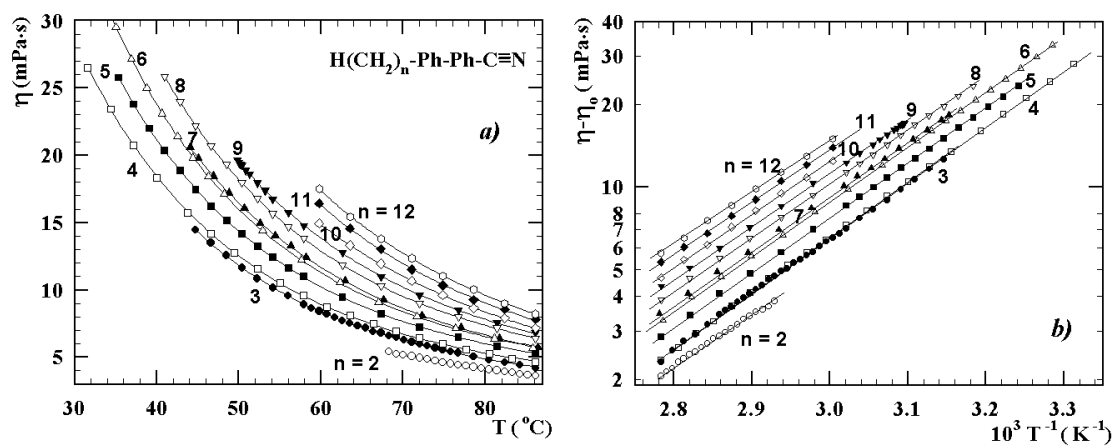

Fig. 2. Temperature dependences of the shear viscosity of $n \mathrm{CB}$ homologous series (a) and Arrhenius-like plots (b) according to Eq. (3).
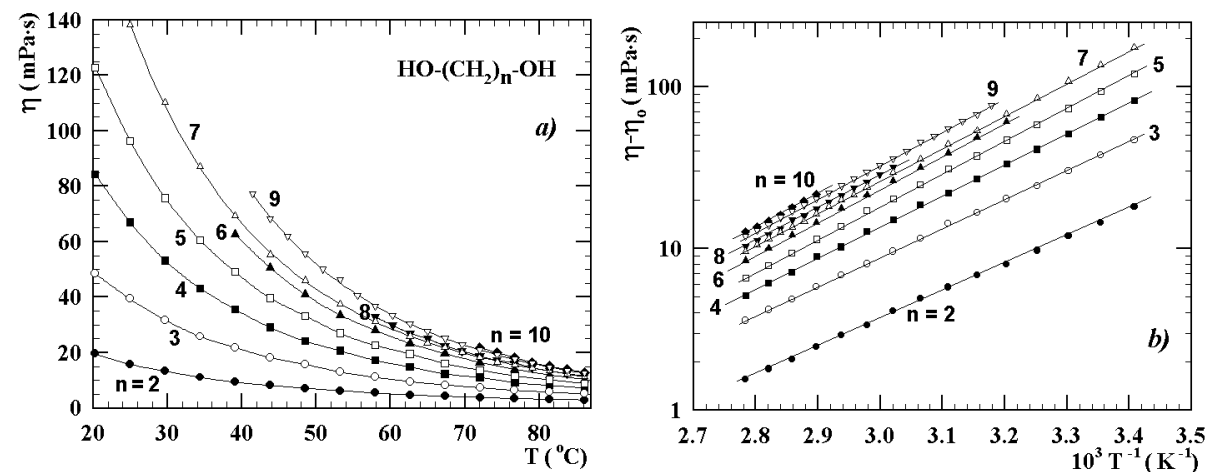

Fig. 3. Temperature dependences of the shear viscosity of $1, n$-alkanediols series (a) and Arrhenius-like plots (b) according to Eq. (3). 


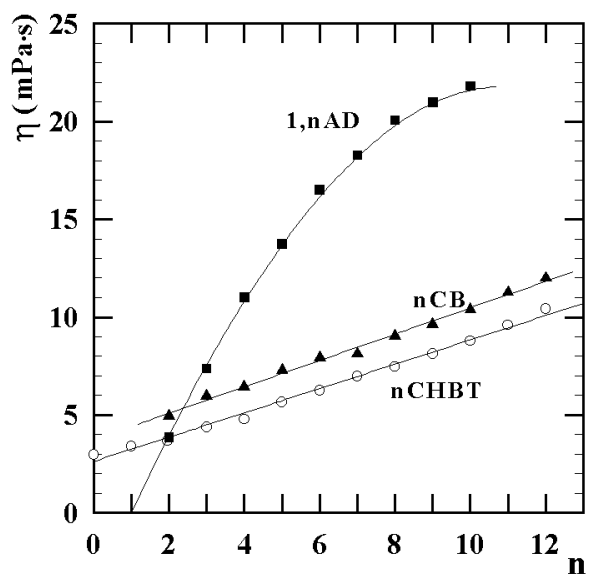

Fig. 4. Isothermal $n$ dependence of the viscosity of studied series of the compounds $\left(T=72^{\circ} \mathrm{C}\right)$.

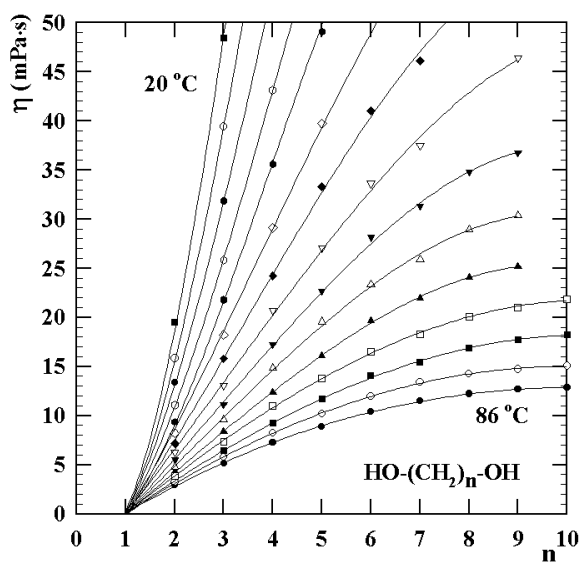

Fig. 5. Isothermal $n$ dependences of the viscosity of $1, n$-alkanediols. The step of temperature is about $5^{\circ} \mathrm{C}$.

It is worth noticing that the extrapolation of experimental $\eta(n)$ dependence of the studied diols to $n=1$ (see Fig. 4) indicates for a very low viscosity of methanediol, $\mathrm{HOCH}_{2} \mathrm{OH}$. Of course, the data do not allow one to estimate the methanediol viscosity value, mainly because of the natural limit of possible changes of $n( \pm 1)$. However, as shown in Fig. 5, all the isothermal viscosities of $1, n$-diols, presented as a function of $n$, show a peculiar behavior concerning the point $n=1$. This fact is so much puzzling, because methanediol is the compound strongly unstable in a pure state and it is quite possible that our data can be useful in understanding that unstability. 
Let us analyze temperature dependence of the measured viscosities. It was shown in many papers that a simple (and friendly) Arrhenius equation

$$
\eta(T)=\eta_{\mathrm{A}} \exp \left(E_{\mathrm{A}} / R T\right)
$$

often does not reproduce satisfactorily the experimental temperature dependence of the viscosity $\eta_{\exp }(T) . E_{\mathrm{A}}$ denotes the viscosity activation energy, $R-$ gas constant, $T$ - absolute temperature and $\eta_{\mathrm{A}}$ - pre-exponential Arrhenius factor. A general rule is: the greater is temperature range of measurements, the greater discrepancy between $\eta_{\exp }(T)$ and prediction of Eq. (1) is observed. However, the discussion on the applicability of Eq. (1) is rather difficult because, in practice, the temperature range $\Delta T$ of the viscosity measurements is very narrow if compared to the whole range of possible $T$ variation $(0, \infty)$; for a typical liquid $\Delta T$ does not exceed $100 \mathrm{~K}$. In such circumstances even a small experimental error can lead to an important discrepancy mentioned above. The simple Arrhenius function (1) with two asymptotes: $\eta=\infty(T=0)$ and $T=\infty(\eta=0)$, and two fitting parameters $\left(E_{\mathrm{A}}\right.$ and $\left.\eta_{\mathrm{A}}\right)$ seems to be too general for description of the real $\eta_{\exp }(T)$ dependences. Nevertheless, Eq. (1) is commonly used in literature mainly because of the activation energy, which even determined with some approximation concerning the linearity of $\ln \eta(T)$ on $T^{-1}$ dependence, is a very useful quantity which can be compared with other activation energies, as obtained from the dielectric relaxation studies, for example.

A more exact portrayal of the experimental viscosity dependence on temperature requires some modifications of the Arrhenius equation (1). In 1920's Vogel and Fulcher (VF) proposed the following equation $[12,13]$ :

$$
\eta(T)=\eta_{\mathrm{VF}} \exp \left(\frac{D T_{0}}{T-T_{0}}\right)
$$

in which three fitting parameters $\left(\eta_{\mathrm{VF}}, D\right.$, and $\left.T_{0}\right)$ occur. Introduction of $T_{0}$ parameter in Eq. (2) formally denotes a shift of the $\eta$-axis from $T=0$ (as for Arrhenius equation) to $T=T_{0}$ and restricts the range of $T$ variation to $\left(T_{0}, \infty\right)$. Although the fitting parameters have no clear physical meaning, Eq. (2) is used with success for empirical description of the viscosity dependence on temperature for many liquids, specially in supercooled state, and some attempts were undertaken in finding the correlations between $T_{0}$ parameter and the vitrification temperature [14-17].

If it were possible to make the $\eta$-axis mobile along the $T$-axis (as in Eq. (2)), the question is why not to make the $T$-axis mobile along the $\eta$-axis? It can be realized in a very simple way by an introduction of a new fitting parameter $\eta_{0}$ as a background in Arrhenius equation (1):

$$
\eta(T)=\eta_{\mathrm{JCL}} \exp \left(E_{\mathrm{A}} / R T\right)+\eta_{0}
$$

The parameter $\eta_{0}$ formally denotes a shift of the $T$-axis from $\eta=0$ (as for Arrhenius equation) to $\eta_{0}$ and restricts the range of $\eta$ variation to $\left(\eta_{0}, \infty\right)$.

Table summarizes the results of the best fitting of Eqs. (1), (2) and (3) to the experimental viscosity data. 
TABLE

Values of the parameters corresponding to the best fitting of Eqs. (1), (2) and (3) to the viscosity experimental data for studied compounds and standard deviation $\sigma$ (Eq. (4)). $n$ denotes the numbers of $-\mathrm{CH}_{2}-$ groups in molecule.

\begin{tabular}{|c|c|c|c|c|c|c|c|c|c|c|c|}
\hline \multirow[b]{2}{*}{$n$} & \multicolumn{3}{|c|}{ Arrhenius (1) } & \multicolumn{4}{|c|}{$\mathrm{VF}(2)$} & \multicolumn{4}{|c|}{ Proposed (3) } \\
\hline & \begin{tabular}{|l|}
$\eta_{\mathrm{A}} \cdot 10^{3}$ \\
$\mathrm{mPa} \cdot \mathrm{s}$
\end{tabular} & \begin{tabular}{|c|}
$E_{\mathrm{A}}$ \\
$\mathrm{kJ} / \mathrm{mol}$
\end{tabular} & $\begin{array}{c}\sigma \\
\mathrm{mPa} \cdot \mathrm{s}\end{array}$ & $\begin{array}{l}\eta_{\mathrm{V}} \cdot 10^{2} \\
\mathrm{mPa} \cdot \mathrm{s}\end{array}$ & $D$ & $\begin{array}{l}T_{0} \\
\mathrm{~K}\end{array}$ & \begin{tabular}{|c|}
$\sigma$ \\
$\mathrm{mPa} \cdot \mathrm{s}$
\end{tabular} & $\begin{array}{c}\eta_{\mathrm{JCL}} \cdot 10^{5} \\
\mathrm{mPa} \cdot \mathrm{s}\end{array}$ & \begin{tabular}{|c|}
$E_{\mathrm{A}}$ \\
$\mathrm{kJ} / \mathrm{mol}$
\end{tabular} & $\begin{array}{c}\eta_{0} \\
\mathrm{mPa} \cdot \mathrm{s}\end{array}$ & $\begin{array}{c}\sigma \\
\mathrm{mPa} \cdot \mathrm{s}\end{array}$ \\
\hline \multicolumn{12}{|c|}{$n$ CHBT } \\
\hline 0 & 1.641 & 21.55 & 0.092 & 12.94 & 3.003 & 176.9 & 0.034 & 11.414 & 27.99 & 1.073 & 0.031 \\
\hline 1 & 1.746 & 21.68 & 0.087 & 11.78 & 3.371 & 172.1 & 0.039 & 15.117 & 27.63 & 1.061 & 0.040 \\
\hline 2 & 1.227 & 22.93 & 0.165 & 12.82 & 3.139 & 178.4 & 0.134 & 7.514 & 29.74 & 1.288 & 0.131 \\
\hline 3 & 1.904 & 22.19 & 0.113 & 36.25 & 1.496 & 215.6 & 0.057 & 4.055 & 31.77 & 1.758 & 0.060 \\
\hline 4 & 0.857 & 24.72 & 0.187 & 20.49 & 2.474 & 193.6 & 0.078 & 3.626 & 32.47 & 1.857 & 0.072 \\
\hline 5 & 2.200 & 22.51 & 0.064 & 7.80 & 6.189 & 141.3 & 0.031 & 3.343 & 33.03 & 2.351 & 0.060 \\
\hline 6 & 1.303 & 24.27 & 0.209 & 45.44 & 1.496 & 219.5 & 0.131 & 3.058 & 33.66 & 2.379 & 0.132 \\
\hline 7 & 1.945 & 23.52 & 0.057 & 18.30 & 3.255 & 182.4 & 0.028 & 3.567 & 33.68 & 2.538 & 0.039 \\
\hline 8 & 1.796 & 23.92 & 0.122 & 31.35 & 2.295 & 200.3 & 0.058 & 8.105 & 31.71 & 2.360 & 0.056 \\
\hline 9 & 1.442 & 24.82 & 0.090 & 17.26 & 3.437 & 182.6 & 0.070 & .494 & 31.74 & 2.138 & 0.069 \\
\hline 10 & 1.496 & 24.92 & 0.134 & 11.95 & 4.722 & 164.6 & 0.122 & 8.416 & 30.22 & 1.941 & 0.120 \\
\hline 11 & 1.097 & 26.08 & 0.180 & 75.92 & 1.122 & 239.4 & 0.111 & 19.971 & 30.43 & 1.635 & 0.151 \\
\hline 12 & 1.378 & 25.64 & 0.169 & 55.12 & 1.644 & 221.4 & 0.095 & & 28.89 & 1.436 & 0.142 \\
\hline \multicolumn{12}{|c|}{$n \mathrm{CB}$} \\
\hline 2 & 1.910 & 22.55 & 0.018 & 10.6 & 3.493 & 180.8 & 0.021 & 1.755 & 34.91 & 1.572 & 0.027 \\
\hline 3 & 243 & 29.00 & 150 & 17.1 & 2.313 & 209.0 & .040 & 771 & 37.80 & 1.886 & 0.051 \\
\hline 4 & 0.104 & 31.53 & 0.435 & 10.2 & 3.537 & 186.3 & 0.053 & 0.622 & 38.45 & 2.302 & 0.068 \\
\hline 5 & 0.150 & 30.86 & 0.331 & 12.4 & 3.336 & 189.9 & 0.057 & 0.890 & 37.91 & 2.424 & 0.036 \\
\hline 6 & 0.094 & 32.42 & 0.453 & 8.9 & 4.100 & 180.6 & .052 & 947 & 38.09 & 2.421 & 0.075 \\
\hline 7 & 0.264 & 29.68 & 0.190 & 9.1 & 4.111 & 180.4 & 0.047 & 1.515 & 36.94 & 2.287 & 0.052 \\
\hline 8 & 0.229 & 30.34 & 0.270 & 11.3 & 3.828 & 184.3 & 0.050 & 1.616 & 37.04 & 2.520 & 0.032 \\
\hline 9 & 0.375 & 29.16 & 0.148 & 15.3 & 3.267 & 193.1 & 0.038 & .166 & 36.47 & 2.481 & 0.036 \\
\hline 10 & 0.629 & 27.88 & 0.038 & 5.3 & 6.338 & 156.7 & 0.042 & 2.361 & 36.49 & 2.474 & 0.080 \\
\hline 11 & 0.526 & 28.63 & 0.120 & 9.8 & 4.368 & 179.7 & 0.084 & 2.500 & 36.63 & 2.505 & 0.075 \\
\hline 12 & 0.544 & 28.71 & 0.073 & 5.4 & 6.442 & 157.5 & 0.043 & 3.278 & 36.09 & 2.487 & 0.059 \\
\hline \multicolumn{12}{|c|}{$1, n \mathrm{AD}$} \\
\hline 2 & 0.326 & 26.76 & 0.29 & 3.035 & 7.178 & 138.9 & 0.12 & 3.305 & 32.22 & 1.396 & 0.11 \\
\hline 3 & 0.130 & 31.26 & 0.41 & 1.282 & 12.05 & 119.2 & 0.19 & 4.058 & 34.05 & 1.566 & 0.20 \\
\hline 4 & 0.073 & 34.02 & 0.61 & 0.849 & 14.74 & 112.8 & 0.20 & 2.658 & 36.45 & 2.108 & 0.26 \\
\hline 5 & 0.043 & 36.26 & 0.75 & 0.420 & 19.39 & 101.7 & 0.24 & 1.848 & 38.27 & 2.337 & 0.32 \\
\hline 6 & 0.054 & 36.22 & 0.28 & 0.282 & 22.04 & 97.5 & 0.12 & 1.906 & 38.87 & 1.968 & 0.15 \\
\hline 7 & 0.041 & 37.25 & 0.64 & 0.129 & 34.50 & 75.0 & 0.30 & 2.422 & 38.53 & 1.884 & 0.29 \\
\hline 8 & 0.045 & 37.16 & 0.13 & 0.041 & 54.24 & 57.0 & 0.12 & 1.806 & 39.57 & 1.237 & 0.11 \\
\hline 9 & 0.033 & 38.34 & 0.35 & 0.019 & 85.89 & 41.1 & 0.35 & 2.449 & 39.11 & 0.703 & 0.35 \\
\hline 10 & 0.025 & 39.30 & 0.12 & 0.005 & 124.15 & 31.5 & 0.14 & 2.069 & 39.77 & 0.224 & 0.13 \\
\hline
\end{tabular}


Standard deviations $\sigma$ of the experimental viscosity $\left(\eta_{\exp }\right)$ from the calculated $\left(\eta_{\text {cal }}\right)$ :

$$
\sigma=\sqrt{\frac{\sum\left(\eta_{\exp }-\eta_{\text {cal }}\right)^{2}}{n_{d}-n_{p}}}
$$

where $n_{d}$ and $n_{p}$ denote the number of the experimental points and the number of the parameters, respectively, are also given in Table. Apart from the compounds for which the measurements were performed (by necessity) in narrow range of temperature, as for $2 \mathrm{CB}$ or 1,10-decanediol, the standard deviation corresponding to Arrhenius equation (1) is assertively higher than that of the remaining two equations.

Equations (2) and (3) reproduce the experimental temperature dependence of the viscosity with the same reliability, so in that field the equations are equivalent. Solid lines in Figs. 1a, 2a, and 3a represent the best fitting obtained with the both equations. However, the strong preponderance of the proposed Eq. (3) is inherent with the fact that the conception of the activation energy is conserved here: it is the slope of $\ln \left(\eta-\eta_{0}\right)$ on $T^{-1}$ dependence.

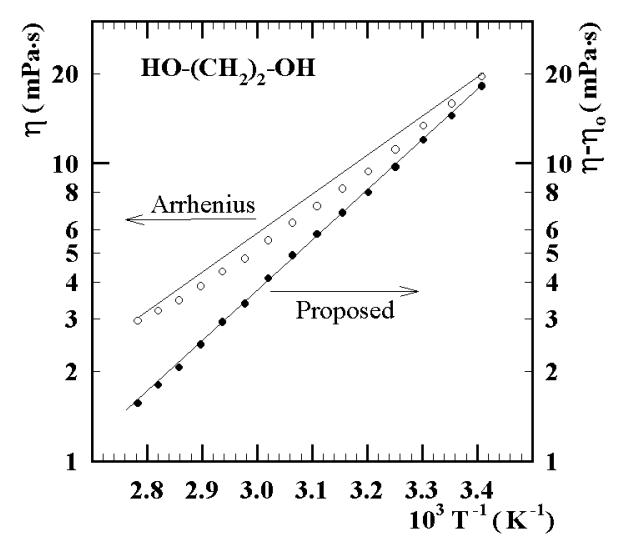

Fig. 6. The experimental viscosity data (open points) measured for 1,2-ethanediol do not fulfill the linear relation predicted by Arrhenius equation (1). Subtraction of the constant value $\eta_{0} \approx 1.4 \mathrm{mPa} \cdot \mathrm{s}$ makes the results (full points) quite well linear, as predicted by Eq. (3).

The essential difference between the plots representing Eqs. (1) and (3) is shown in Fig. 6. It is clear that the apparent slop of the Arrhenius plot depends on the range of temperature studied, because the $\ln \eta$ on $T^{-1}$ dependence is not linear. Still, if one takes into account the background, i.e. one subtracts the constant $\eta_{0}$ from the experimental viscosity, the obtained dependence $\ln \left(\eta-\eta_{0}\right)$ on $T^{-1}$ is quite good linear and the activation energy can be determined here quite univocally. Now, the question is about the physical meaning of the background $\eta_{0}$. It is possible that $\eta_{0}$ represents an effective residual viscosity resulting from the experimental 
imperfections in the viscosity measurements, which can appear, for example, in the proximity of the edges of rotating parts of the measuring system or from other similar effects.

Figures $1 \mathrm{~b}, 2 \mathrm{~b}$, and $3 \mathrm{~b}$ show that the linearity forecasting by Eq. (3) is well fulfilled for all studied liquids. The viscosity activation energy for studied series of compounds, derived from the slope of the lines, are presented in Fig. 7, as a function of the $n$ number.

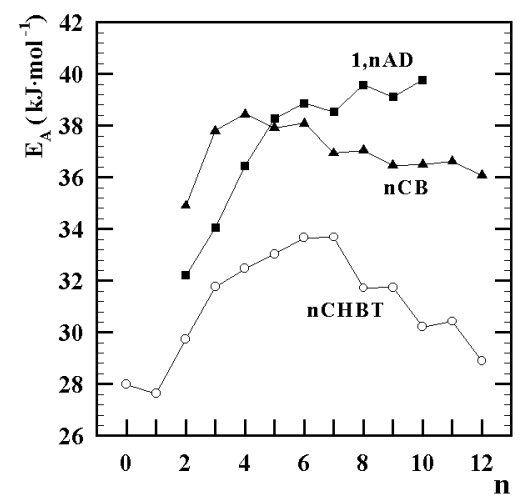

Fig. 7. $n$ dependence of the viscosity activation energy corresponding to the best fitting of Eq. (3) to the experimental viscosity data for studied compounds.

The following conclusion results from the analysis of Fig. 7: temperature dependence of the viscosity of studied low-molecular weight liquids, which can be represented by the viscosity activation energy, shows only a weak relation to the type and strength of the intermolecular interactions and to the length of the molecules. Despite of quite great differences in the viscosity values, as for example between $n \mathrm{CB}$ and $1, n$-diols, the lines $\ln \left(\eta-\eta_{0}\right)$ vs. $T^{-1}$ are practically parallel to each other, i.e. the viscosity activation energies are close to each other. The conclusion seems to be important in the searching of liquids with a weak temperature dependence of the viscosity.

\section{References}

[1] D.J. Evans, Mol. Phys. 34, 103, 161 (1977).

[2] S. Hess, H.J.M. Hanley, Phys Lett. A 98, 35 (1983).

[3] H.-M. Koo, S. Hess, Physica A 145, 361 (1987).

[4] R. Edberg, G.P. Morriss, D.J. Evans, J. Chem. Phys. 86, 4555 (1987).

[5] C. Hoheisel, J. Chem. Phys. 86, 2328 (1987).

[6] D. Levesque, L. Verlet, Mol. Phys. 61, 143 (1987).

[7] G. Maréchal, J.-P. Ryckaert, A. Bellemans, Mol. Phys. 61, 33 (1987). 
[8] R. Vogelsang, G. Hoheisel, M. Lucas, Mol. Phys. 64, 1203 (1988).

[9] S. Sarman, J. Chem. Phys. 103, 10378 (1995).

[10] W. Allen, R. Rowley, J. Chem. Phys. 106, 10273 (1997).

[11] J. Jadżyn, R. Dąbrowski, K. Glumiak, G. Czechowski, J. Chem. Eng. Data 45, 1027 (2000); J. Jadżyn, R. Dąbrowski, T. Lech, G. Czechowski, J. Chem. Eng. Data 46, 110 (2001); T. Lech, G. Czechowski, J. Jadżyn, J. Chem. Eng. Data 46, 725 (2001).

[12] H. Vogel, Phys. Z. 22, 645 (1921).

[13] G.S. Fulcher, J. Am. Ceram. Soc. 8, 339 (1923).

[14] C.A. Angell, J. Non-Cryst. Solids 102, 205 (1988).

[15] L.R. Cook, H.E. King, Jr., C.A. Herbst, D.R. Herschbach, J. Chem. Phys. 100, 5178 (1994).

[16] R. Böhmer, K.L. Ngai, C.A. Angell, D.J. Plazek, J. Chem. Phys. 99, 4201 (1993).

[17] D. Shenoy, S. Filippov, F. Alier, P. Keller, T. Thomsen, B. Rata, Phys. Rev. E 62, $8100(2000)$. 